\title{
The Effect of Normal Contact Forces on the Stress in Shear Rate Invariant Particle Suspensions
}

\author{
J. J. J. Gillissen and H. J. Wilson \\ Department of Mathematics, University College London, \\ Gower Street, London, WC1E 6BT. \\ E-mail: jurriaangillissen@gmail.com
}

(Dated: December 22, 2018)

\begin{abstract}
We present a tensorial theory for the microstructure and the stress in shear rate invariant particle suspensions, that includes hydrodynamic and normal but not tangential hard sphere interaction forces. The theory predicts, that hydrodynamic forces produce a negligible first normal stress difference, while contact forces produce a positive first normal stress difference. The theory thereby provides a rationale for seemingly contradicting experimental observations in the literature. In addition, the theory captures experimentally observed time dependence of the shear stress after shear reversal.
\end{abstract}




\section{${ }_{8}$ I. INTRODUCTION}

9 Particle suspensions occur ubiquitously in nature, and their mechanical stress $\boldsymbol{\Sigma}$ is gov10 erned by the particle interaction forces, which can be classified into hydrodynamic, and non11 hydrodynamic. The nature of the hydrodynamic forces depends on the particle Reynolds 12 number $\operatorname{Re}_{p}=\dot{\gamma} a^{2} / \nu$, where $a$ is the particle radius, $\nu=\eta / \rho$ is the solvent kinematic vis13 cosity, $\eta$ is the solvent dynamic viscosity, $\rho$ is the solvent mass density, and $\dot{\gamma}$ is the shear ${ }_{14}$ rate. When: $\operatorname{Re}_{p} \ll 1$, flow inertia is negligible, and the hydrodynamic forces are governed 15 by the linear Stokes equations.

16 The Stokes equations predict that particles make no physical contacts in a fluid, since 17 the lubrication force diverges at contact [1]. With increasing volume fraction $\phi$ however, 18 the lubrication films become progressively thinner, and when their thickness approaches the 19 atomic length scale, the films disintegrate, resulting in physical contacts.

20 This work addresses the effect of hard and frictionless contact forces on the particle stress. ${ }_{21}$ Hard contacts do not introduce a force scale $F$ into the system, and the non-dimensional 22 suspension viscosity: $\Sigma_{12} / \eta \dot{\gamma}$, depends therefore only on the particle volume fraction $\phi$ and 23 not on the shear rate $\dot{\gamma}$, as this can not be non-dimensionalised into $a^{2} \eta \dot{\gamma} / F$, due to the 24 absence of $F$. This study is therefore restricted to shear rate invariant suspensions.

25 Experimental data on the suspension stress $\boldsymbol{\Sigma}$ are mainly concerned with shear flow, 26 where: $\boldsymbol{L}=\boldsymbol{\nabla} \boldsymbol{U}^{T}=\dot{\gamma} \boldsymbol{\delta}_{1} \boldsymbol{\delta}_{2}$ is the velocity gradient tensor, $\dot{\gamma}=\sqrt{2 \boldsymbol{E}: \boldsymbol{E}}$ is the shear rate, ${ }_{27} \boldsymbol{E}=\frac{1}{2}\left(\boldsymbol{L}+\boldsymbol{L}^{T}\right)$ is the strain rate tensor, $\boldsymbol{U}$ is the velocity vector, and 1,2 and 3 are the ${ }_{28}$ flow direction, the gradient direction and the vorticity direction, respectively

29 Fig. 1 summarises experimental data on the relative, first and second normal stress 30 differences in shear rate invariant suspensions. These quantities are defined as: $\zeta_{1}=\left(\Sigma_{11}-\right.$ $\left.{ }_{31} \Sigma_{22}\right) / \Sigma_{12}$, and: $\zeta_{2}=\left(\Sigma_{22}-\Sigma_{33}\right) / \Sigma_{12}$, respectively. While $\zeta_{2}$ has always been observed to 32 be negative, $\zeta_{1}$ has been observed to be both negative and small (compared to $\zeta_{2}$ ) [5-7], 33 as well as positive $[2,4]$. It is noted that a positive $\zeta_{1}$ has also been observed in shear 34 thickening suspensions [8-10], which supports the hypothesis that particle contact forces are 35 responsible for $\zeta_{1}>0$ [11].

36 In addition to normal stresses, effects of contact forces are also reflected by a stress dis37 continuity upon the reversal of shear flow. In the absence of contacts, the Stokes equation 38 dictates, that the stress is linear in the velocity. This means, that, when the flow velocity 




FIG. 1. Measured, steady, relative, first and second normal stress differences: $\zeta_{1}=\left(\Sigma_{11}-\Sigma_{22}\right) / \Sigma_{12}$ (open markers), and: $\zeta_{2}=\left(\Sigma_{22}-\Sigma_{33}\right) / \Sigma_{12}$ (filled markers), under shear rate invariant conditions, and as functions of the particle volume fraction $\phi . \triangle: a=20 \mu \mathrm{m}, \nabla: a=70 \mu \mathrm{m}$ polystyrene in water, UCON oil and zinc bromide [2]; $\square: a=35 \mu \mathrm{m}, \diamond: a=70 \mu \mathrm{m}$ polystyrene in poly(ethylene glycol-ran-propylene glycol) monobutylether [3]; $\triangleleft: a=5 \mu$ m poly (methyl methacrylate) (PMMA) in Triton X-100, anhydrous zinc chloride, and water (TZW) [4]; $\oplus a=98 \mu \mathrm{m}$ PMMA in TZW [5]; $\bigcirc: a=22 \mu \mathrm{m}$ glass in corn syrup and glycerin [6]; 项 $a=20 \mu \mathrm{m}$ polystyrene in silicone fluid [7]. The lines are drawn to guide the eye, and the lower line represents the empirical relation [Eq. $(16)]$.

39 is instantaneously reversed, the stress is instantaneously reversed too, as observed experi40 mentally for small $\phi[12]$. For large $\phi$, particles may experience contacts, and since contact ${ }_{41}$ forces are not reversed upon flow reversal, there is a discontinuity in the (absolute value of 42 the) particle stress upon flow reversal [12-14].

43 In this work we provide a micro-structural explanation for the above mentioned experi44 mental observations, regarding normal stresses in steady shear flow, and stress discontinuity 45 after shear reversal. To this end we include hard and frictionless contact forces into a ${ }_{46}$ previously proposed tensorial theory for the suspension microstructure and stress [15]. 


\section{${ }_{47}$ II. DERIVATION OF THE THEORY}

48

49 First we summarise the theory in the absence of contact forces. For a full derivation, the 50 reader is referred to Ref. [15]. In the two-body approximation the stress is given by [16-19]:

$$
\boldsymbol{\Sigma}=-\frac{1}{V} \sum_{\alpha>\beta} \boldsymbol{F}_{\alpha, \beta} \boldsymbol{r}_{\alpha, \beta}=-n\langle\boldsymbol{F} \boldsymbol{r}\rangle .
$$

${ }_{51}$ Here: $n=N / V$, is the particle number density, $N$ is the number of particles inside the 52 averaging volume $V, \boldsymbol{F}_{\alpha, \beta}$ is the interaction force $\boldsymbol{F}$ between particles $\alpha$ and $\beta$, and $\boldsymbol{r}_{\alpha, \beta}$ ${ }_{53}$ is the corresponding particle pair separation vector $\boldsymbol{r}=\boldsymbol{p} r$, where $\boldsymbol{p}$ is the particle pair ${ }_{54}$ orientation unit vector, and $r=|\boldsymbol{r}|$ is the particle pair separation. The stress is dominated 55 by particles with small gaps:

$$
\boldsymbol{r}=2 a \boldsymbol{p}
$$

${ }_{56}$ The interaction force $\boldsymbol{F}$ :

$$
\boldsymbol{F}=\boldsymbol{F}_{h}+\boldsymbol{F}_{c}
$$

57 is the sum of the hydrodynamic force $\boldsymbol{F}_{h}$, and the contact force $\boldsymbol{F}_{c}$, which is assumed zero, ${ }_{58}$ for the moment. The pair separation vector evolves as:

$$
\dot{\boldsymbol{r}}=c_{1} \boldsymbol{L}: \boldsymbol{r p p}+\boldsymbol{L} \cdot \boldsymbol{r} \cdot(\boldsymbol{\delta}-\boldsymbol{p} \boldsymbol{p})
$$

59 and the corresponding lubrication force is to leading order:

$$
\boldsymbol{F}_{h}=-a^{2} \eta c_{2} \boldsymbol{E}: \boldsymbol{p p p}
$$

${ }_{60}$ Here $c_{1}$ and $c_{2}$ are non-dimensional functions of $r / a$ and $\phi$. Combining Eqs. $(1,2,3,5)$ and 61 using that $\phi \sim n a^{3}$ gives the following particle stress tensor:

$$
\Sigma=\alpha \eta \boldsymbol{E}:\langle\boldsymbol{p} \boldsymbol{p} \boldsymbol{p} \boldsymbol{p}\rangle
$$

${ }_{62}$ Here $\alpha=\tilde{c}_{2} \phi$ is the lubrication parameter, and $\tilde{c}_{2}$ is the effective $c_{2}$, which is averaged over ${ }_{63}$ the distribution of pair configurations, and which diverges when $\phi$ approaches maximum ${ }_{64}$ packing. 
The average $\langle\cdots\rangle$ in Eq. (6) is expressed as an integral over the probability distribution ${ }_{66}$ function $\Psi(\boldsymbol{r})$ of the particle pair separation vector $\boldsymbol{r}$ :

$$
\langle\cdots\rangle=\int_{|\boldsymbol{r}|=2 a}^{|\boldsymbol{r}|=2 a+\delta r} \Psi(\boldsymbol{r}) \cdots d^{3} \boldsymbol{r},
$$

${ }_{67}$ where the integration is restricted to the so-called 'interaction shell', where particle pairs ${ }_{68}$ have small gaps: $0<r-2 a<\delta r$. The evolution of $\Psi(\boldsymbol{r})$ is governed by the Smoluchowski 69 equation:

$$
\partial_{t} \Psi+\partial_{k}\left(\dot{r}_{k} \Psi\right)=0
$$

Since computing $\Psi(\boldsymbol{r})$ is costly, we compute instead its second order orientation moment $\boldsymbol{a}=\langle\boldsymbol{p} \boldsymbol{p}\rangle$, referred to as the microstructure. The evolution equation for $\boldsymbol{a}$ is derived, by inserting Eq. (4) into Eq. (8), multiplying the result by $\boldsymbol{p p}$, and integrating the result from $r=2 a$ to $r=2 a+\delta r$; see Ref. [15]:

$$
\begin{aligned}
& \partial_{t}\langle\boldsymbol{p} \boldsymbol{p}\rangle=\boldsymbol{L} \cdot\langle\boldsymbol{p} \boldsymbol{p}\rangle+\langle\boldsymbol{p} \boldsymbol{p}\rangle \cdot \boldsymbol{L}^{T}-2 \boldsymbol{L}:\langle\boldsymbol{p} \boldsymbol{p} \boldsymbol{p} \boldsymbol{p}\rangle \\
&-\beta\left[\boldsymbol{E}_{e}:\langle\boldsymbol{p} \boldsymbol{p} \boldsymbol{p} \boldsymbol{p}\rangle+\frac{1}{15}\left(2 \boldsymbol{E}_{c}+\operatorname{Tr}\left(\boldsymbol{E}_{c}\right) \boldsymbol{\delta}\right)\right] .
\end{aligned}
$$

70 The first line of Eq. (9) described rotation of rigid dumbbells, i.e. fixed pair separations. The 71 second line accounts for changes in the separation, which correspond to an orientation flux 72 between the interaction shell and the exterior. This term is interpreted as the association and 73 dissociation of interacting particle pairs, by the action of the compressive and the extensional ${ }_{74}$ parts of the rate of strain tensor: $\boldsymbol{E}_{c}$ and $\boldsymbol{E}_{e}$, respectively, which pushes particles together 75 and pulls them apart, respectively. These effects are controlled by the pair association rate $76 \beta$, which is an increasing function of $\phi$.

To close the theory a relation is needed to express the fourth order moment $\langle\boldsymbol{p} \boldsymbol{p} \boldsymbol{p} \boldsymbol{p}\rangle$ in terms of the second order moment $\langle\boldsymbol{p} \boldsymbol{p}\rangle$. Here we use the linear closure, that was proposed in Ref. [20], which is accurate when the distribution is close to isotropy, such that $\Psi(\boldsymbol{p})$ is well captured by a linear expansion in the anisotropy tensor $\boldsymbol{a}-\boldsymbol{\delta} / 3$, i.e.: $\Psi(\boldsymbol{p})=$

$$
\begin{aligned}
& (4 \pi)^{-1}\left[1+\frac{15}{2}(\boldsymbol{a}-\boldsymbol{\delta} / 3): \boldsymbol{p} \boldsymbol{p}\right] . \\
& \left\langle p_{i} p_{j} p_{k} p_{l}\right\rangle=-\frac{1}{35}\left\langle p_{m} p_{m}\right\rangle\left(\delta_{i j} \delta_{k l}+\delta_{i k} \delta_{j l}+\delta_{i l} \delta_{j k}\right) \\
& +\frac{1}{7}\left(\delta_{i j}\left\langle p_{k} p_{l}\right\rangle+\delta_{i k}\left\langle p_{j} p_{l}\right\rangle+\delta_{i l}\left\langle p_{j} p_{k}\right\rangle+\left\langle p_{i} p_{j}\right\rangle \delta_{k l}+\left\langle p_{i} p_{k}\right\rangle \delta_{j l}+\left\langle p_{i} p_{l}\right\rangle \delta_{j k}\right) .
\end{aligned}
$$


Here, we extend the theory with hard and frictionless contact forces. We consider a 79 limiting member of the class of shear rate invariant suspensions in which the interparticle 80 friction coefficient vanishes and tangential friction forces may be ignored; see e.g. Ref. [21], 81 and the microstructure equation [Eq. (9)] is unaffected by the contact forces. It is noted, 82 that under shear thickening conditions, the tangential friction may have an effect on the 8з particle motion, involving a transition from sliding to rolling friction, and these effects are 84 not captured by the present theory.

85 By definition, the normal contact force $\boldsymbol{F}_{c}$ is directed along $\boldsymbol{p}$, i.e. $\boldsymbol{F}_{c}=\left|\boldsymbol{F _ { c }}\right| \boldsymbol{p}$ when a ${ }_{86}$ particle pair is under compression, while it is zero, when a pair is under extension. The 87 contact force magnitude $\left|\boldsymbol{F}_{c}\right|$ is therefore assumed to be proportional to the compressive 88 part $\boldsymbol{E}_{c}$ of $\boldsymbol{E}$ projected onto $\boldsymbol{p}$, i.e. $\left|\boldsymbol{F}_{c}\right|=-c_{3} a^{2} \eta \boldsymbol{E}_{c}: \boldsymbol{p} \boldsymbol{p}$, where $c_{3}$ is a non-dimensional 89 function of $\boldsymbol{p}$, and $a^{2} \eta$ is added to make the expression dimensionally correct.

$$
\boldsymbol{F}_{c}=-c_{3} a^{2} \eta \boldsymbol{E}_{c}: \boldsymbol{p} \boldsymbol{p p} .
$$

${ }_{90}$ Combining Eqs. $(1,2,3,5,11)$ and using that $\phi \sim n a^{3}$ we arrive at the following particle 91 stress tensor:

$$
\boldsymbol{\Sigma}=\eta\left(\alpha \boldsymbol{E}+\chi \boldsymbol{E}_{c}\right):\langle\boldsymbol{p} \boldsymbol{p} \boldsymbol{p} \boldsymbol{p}\rangle,
$$

92 where $\chi=\tilde{c}_{3} \phi$ is the contact parameter, and $\tilde{c}_{3}$ is the effective $c_{3}$, which is averaged over ${ }_{93}$ the distribution of pair configurations, and which diverges when $\phi$ approaches maximum 94 packing.

\section{III. THEORETICAL PREDICTIONS}

\section{$96 \quad$ A. Steady Shear}

In shear flow Eqs. $(9,10)$ predict that particle pairs associate in the compressive quadrants: $a_{12}<0$, rotate towards $x_{2}$, and dissociate in the extensional quadrants $a_{12}>0$. For: $\beta>3$, the association and dissociation dominate the rotation. The resulting distribution aligns in the compressive quadrant: $a_{12}<0$, with a slight tilt towards $x_{2}$, i.e.: $a_{22}>a_{11}$. For: $\beta<3$, on the other hand, the pair rotation dominates the association and dissociation. Starting from isotropy, the resulting distribution oscillates and dampens towards a preferred 

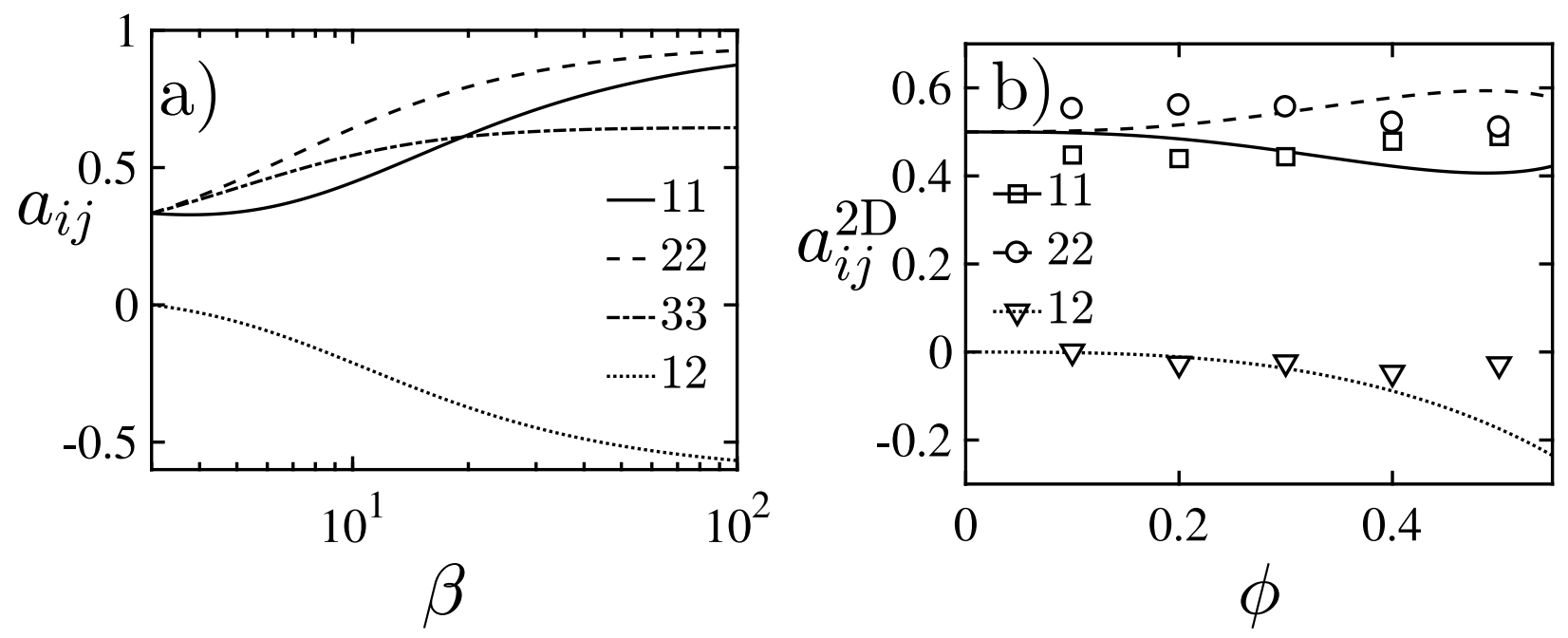

FIG. 2. (a) Modelled [Eqs. $(9,10)]$ microstructure $\boldsymbol{a}$ as a function of the pair association rate $\beta$. (b) Volume fraction $\phi$ dependence of the planar microstructure $\boldsymbol{a}^{2 \mathrm{D}}$, measured in Ref. [22] [Eq. (14), markers] and modelled [Eqs. (13, 15, 16), lines].

alignment in the $x_{1}$ direction, corresponding to $a_{11}>a_{22}$ and $a_{12}>0$. As these oscillations have not been observed in experiments, we restrict the following analysis to $\beta>3$. The corresponding analytical solution to Eqs. (9, 10):

$$
\begin{aligned}
\boldsymbol{a}=(6240+ & \left.810 \beta+135 \beta^{2}\right)^{-1} \\
& \times\left(\begin{array}{ccc}
3256-374 \beta+129 \beta^{2} & -252 \beta+84 \beta^{2} & 0 \\
-252 \beta+84 \beta^{2} & 904+410 \beta+129 \beta^{2} & 0 \\
0 & 0 & 820+564 \beta+87 \beta^{2}
\end{array}\right),
\end{aligned}
$$

97 is plotted as a function of $\beta$ in Fig. 2a.

98 In Fig. 2b, we compare the modelled microstructure to experimental data from Ref. [22], 99 reporting the planar, pair distribution function $\Psi^{2 \mathrm{D}}$ in the $\left(r_{1}, r_{2}\right)$ - plane. In Fig. $2 \mathrm{~b}$ the 100 markers indicate the corresponding, measured, planar moments $\boldsymbol{a}^{2 \mathrm{D}}$ :

$$
\boldsymbol{a}^{2 \mathrm{D}}=\int_{|\boldsymbol{r}|=1.7 a}^{|\boldsymbol{r}|=2.3 a} \Psi^{2 \mathrm{D}}(\boldsymbol{r}) \boldsymbol{p} \boldsymbol{p} d^{2} \boldsymbol{r} .
$$

101 These measurement data show a weak departure from isotropy over the entire $\phi$-range, which 102 supports the validity of the linear closure [Eq. (10)]. To compare our theory [Eq. (13)] to 

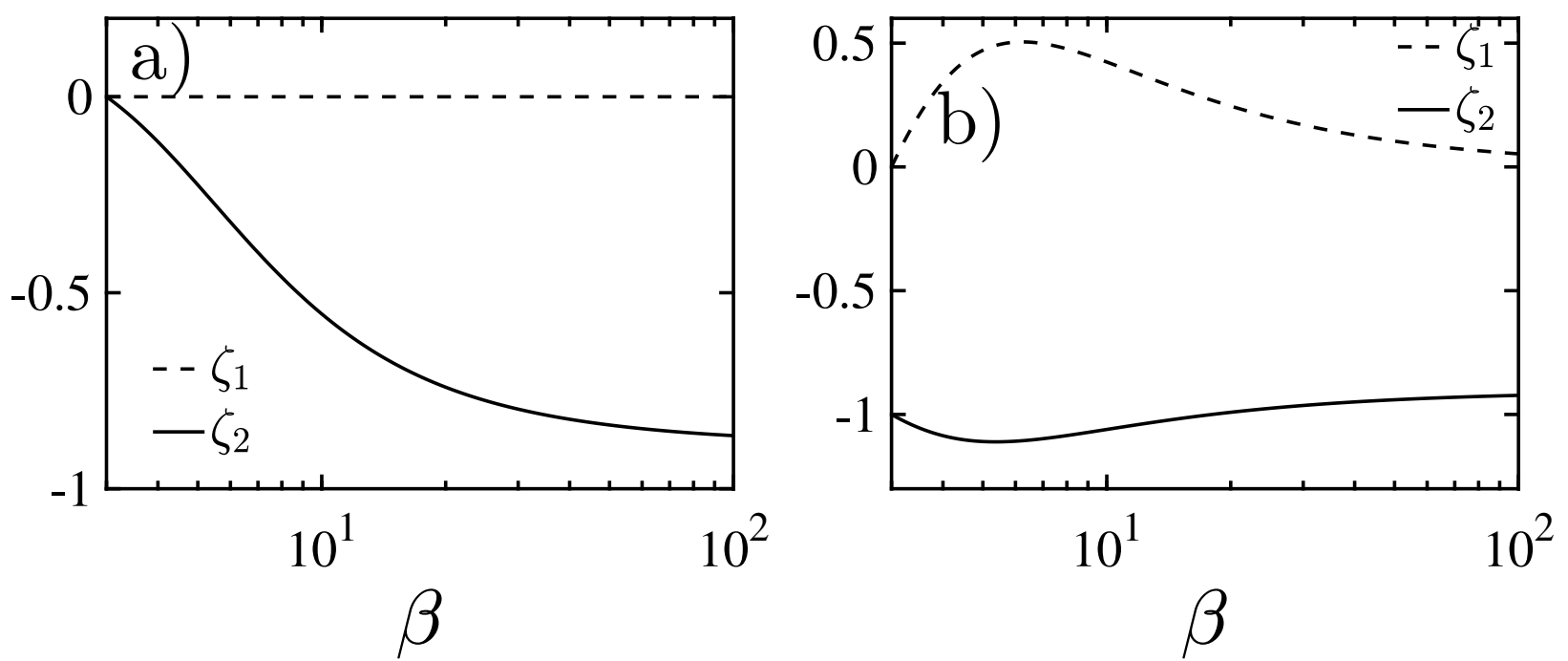

FIG. 3. Modelled [Eqs. $(9,10,12)]$ relative, first normal stress difference: $\zeta_{1}=\left(\Sigma_{11}-\Sigma_{22}\right) / \Sigma_{12}$ (dashed lines), and second normal stress difference: $\zeta_{2}=\left(\Sigma_{22}-\Sigma_{33}\right) / \Sigma_{12}$ (solid lines), as functions of the pair association rate $\beta$ for systems dominated by (a) hydrodynamic forces: $(\alpha, \chi)=(1,0)$ and $(b)$ contact forces $(\alpha, \chi)=(0,1)$.

103 these experimental data, we convert the volumetric moments $\boldsymbol{a}$ into the planar moments ${ }_{104} \boldsymbol{a}^{2 \mathrm{D}}$ using:

$$
\boldsymbol{a}^{2 \mathrm{D}}=\frac{\boldsymbol{a}}{a_{11}+a_{22}}
$$

105 and we convert $\beta$ to $\phi$ by using the modelled relation between $\beta$ and $\zeta_{2}$ [see Eq. (17) below, 106 assuming $\chi=0]$, and the empirical relation:

$$
\zeta_{2}=-4 \phi^{3}
$$

107 which captures the experimental data shown in Fig. 1. In Fig. 2b the resulting modelled ${ }_{108} \boldsymbol{a}^{2 \mathrm{D}}$ are plotted with the lines. Both experimental data and theory predict that: $a_{12}^{2 \mathrm{D}}<0$, 109 and: $a_{22}^{2 \mathrm{D}}>a_{11}^{2 \mathrm{D}}$.

110 The relative, first and second normal stress differences are obtained by inserting Eq. (13) 111 into Eqs. (10, 12), giving:

$$
\left(\begin{array}{l}
\zeta_{1} \\
\zeta_{2}
\end{array}\right)=\left(\begin{array}{c}
\frac{336(\beta-3) \chi}{\alpha\left(54 \beta^{2}-24 \beta+904\right)+\left(63 \beta^{2}-120 \beta+452\right) \chi} \\
-\frac{48 \alpha(\beta-3) \beta+\left(57 \beta^{2}+6 \beta+128\right) \chi}{\alpha\left(54 \beta^{2}-24 \beta+904\right)+\left(63 \beta^{2}-120 \beta+452\right) \chi}
\end{array}\right),
$$


112 which are plotted as functions of $\beta$ for contactless systems: $(\alpha, \chi)=(1,0)$, in Fig. 3a, and 113 for contact dominated systems: $(\alpha, \chi)=(0,1)$, in Fig. 3b. For systems without contact ${ }_{114}$ forces, we find: $\zeta_{1}=0$ and $\zeta_{2}<0$ (Fig. 3a), and for systems with contact forces we find: ${ }_{115} \zeta_{1}>0$ and $\zeta_{2}<0$ (Fig. 3b). These results may rationalise the data shown in Fig. 1, 116 and suggest, that the stress in Refs. [5-7] is dominated by hydrodynamic forces, while 117 that in Refs. $[2,4]$ is dominated by contact forces. It is further noted that, the positive ${ }_{118}$ effect of contact forces on the first normal stress difference in shear invariant suspensions, is 119 supported by two dimensional Stokesian Dynamics simulations in Ref. [23].

${ }_{120}$ The transition from negligible to significantly positive $\zeta_{1}$ is explained as follows. The 121 hydrodynamic part of the particle stress [Eq. (12)] produces a first normal stress difference:

$$
\Sigma_{11}-\Sigma_{22}=\alpha \eta \dot{\gamma}\left(\left\langle p_{1} p_{1} p_{1} p_{2}\right\rangle-\left\langle p_{2} p_{2} p_{2} p_{1}\right\rangle\right)
$$

122 which is quadratic in the microstructure anisotropy, and is therefore ignored by the linear ${ }_{123}$ closure [Eq. (10)], which predicts $\zeta_{1}=0$. The contact part of the particle stress, on the 124 other hand, produces a first normal stress difference:

$$
\Sigma_{11}-\Sigma_{22}=\chi \eta \dot{\gamma}\left[\frac{1}{2}\left\langle p_{1} p_{1} p_{1} p_{2}\right\rangle-\frac{1}{2}\left\langle p_{2} p_{2} p_{2} p_{1}\right\rangle+\frac{1}{4}\left\langle p_{2} p_{2} p_{2} p_{2}\right\rangle-\frac{1}{4}\left\langle p_{1} p_{1} p_{1} p_{1}\right\rangle\right]
$$

125 which is first order in the microstructure anisotropy, and according to the linear closure [Eq. $126(10)]$ :

$$
\Sigma_{11}-\Sigma_{22}=\frac{3}{14} \chi \eta \dot{\gamma}\left(a_{22}-a_{11}\right)
$$

127 which is positive, since $a_{22}>a_{11}$; see Fig. $2 \mathrm{~b}$.

\section{$128 \quad$ B. Shear Reversal}

${ }_{129}$ Finally we consider the case of shear reversal. We use the Euler forward integration 130 scheme with a time step of: $\Delta t=0.01 / \dot{\gamma}$, to compute the time dependent microstructure 131 and stress after shear reversal, using various values for $\beta, \alpha$ and $\chi$. In the computation, 132 the initially isotropic suspension: $\boldsymbol{a}=\boldsymbol{\delta} / 3$, is sheared until a steady state is reached, after 133 which the flow direction is reversed from negative to positive, at which instant we define: ${ }_{134} t=0$. The reversal induces a reorganisation of the microstructure and the attainment of a 135 new steady state. 

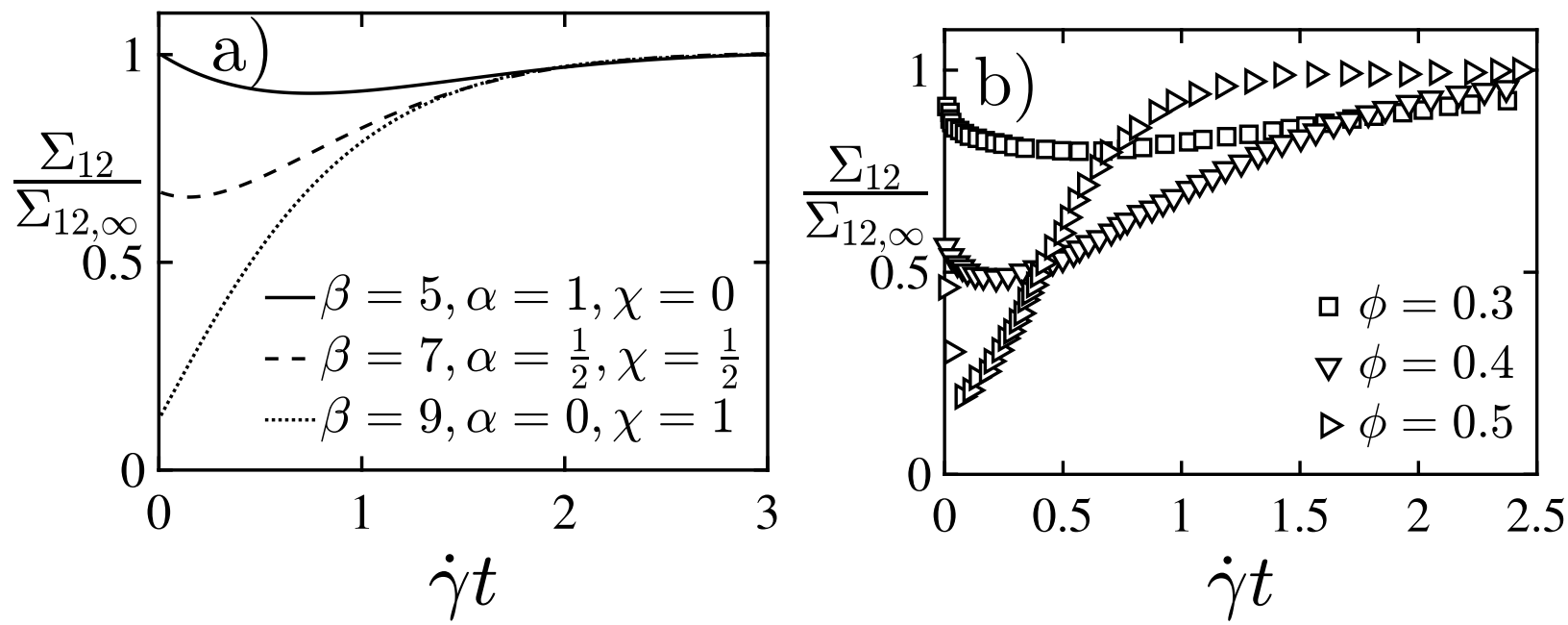

FIG. 4. (a) Modelled [Eqs. (9, 10, 12)] suspension shear stress, scaled with the steady value: $\Sigma_{12} / \Sigma_{12, \infty}$, as a function of the strain $\dot{\gamma} t$, after shear reversal, using various $\beta, \alpha, \chi$. (b) Measured $\Sigma_{12} / \Sigma_{12, \infty}$ as a function of $\dot{\gamma} t$, after shear reversal, using various volume fractions $\phi$ [12].

136 The modelled shear stress, scaled with the steady value $\Sigma_{12} / \Sigma_{12, \infty}$ is plotted as a function ${ }_{137}$ of the strain $\dot{\gamma} t$ in Fig. 4a. As expected, the stress in the contactless theory: $(\alpha, \chi)=(1,0)$, 138 is conserved, upon shear reversal, followed by a decrease and subsequent recovery to the 139 steady value. In the contact dominated theory: $(\alpha, \chi)=(0,1)$, on the other hand, the shear 140 stress is not conserved upon shear reversal, i.e., there is a discontinuous drop, followed by 141 a recovery, in qualitative agreement with experimental data from literature [12], which are ${ }_{142}$ plotted in Fig. 4b. The qualitatively correct prediction of the stress discontinuity, which 143 is related to the contact forces, further validates the physical significance of the proposed 144 constitutive equations [Eqs. $(9,10,12)]$.

\section{${ }_{145}$ IV. CONCLUSION}

We propose a tensorial theory for suspension microstructure and stress, that includes 147 both hydrodynamic and hard sphere interaction forces.

148 The theory assumes hard and frictionless contact forces, which is a reasonable assumption 149 for shear rate invariant suspensions, but may not be valid for shear thickening suspensions. ${ }_{150}$ The theory furthermore assumes a linear relationship between the stress and the microstruc- 
151 ture anisotropy [Eq. (10)], which is supported by experimental data in the literature [22], 152 as illustrated in Fig. 2b.

${ }_{153}$ The theory predicts that hydrodynamic forces produce a negligible first normal stress ${ }_{154}$ difference $\zeta_{1}$, while contact forces produce a positive $\zeta_{1}$. These results may provide a rationale 155 for seemingly contradicting experimental observations in the literature, as illustrated in Fig. 1561.

\section{ACKNOWLEDGMENTS}

We would like to acknowledge financial support from the Engineering and Physical Sciences Research Council of the United Kingdom Grant No. EP/N024915/1.

[1] S Kim and S Karilla, Microhydrodynamics (Butterworth-Heinemann Publish, 1991).

[2] Talib Dbouk, Laurent Lobry, and Elisabeth Lemaire, "Normal stresses in concentrated nonBrownian suspensions," J. Fluid Mech. 715, 239-272 (2013).

[3] Étienne Couturier, François Boyer, Olivier Pouliquen, and Élisabeth Guazzelli, "Suspensions in a tilted trough: second normal stress difference," J. Fluid Mech. 686, 26-39 (2011).

[4] Chaiwut Gamonpilas, Jeffrey F Morris, and Morton M Denn, "Shear and normal stress measurements in non-brownian monodisperse and bidisperse suspensions," J. Rheol. 60, 289296 (2016).

[5] Anugrah Singh and Prabhu R Nott, "Experimental measurements of the normal stresses in sheared Stokesian suspensions," J. Fluid Mech. 490, 293-320 (2003).

[6] Isidro E Zarraga, Davide A Hill, and David T Leighton Jr, "The characterization of the total stress of concentrated suspensions of noncolloidal spheres in Newtonian fluids," J. Rheol. 44, $185-220(2000)$.

[7] Shao-Cong Dai, Erwan Bertevas, Fuzhong Qi, and Roger I Tanner, "Viscometric functions for noncolloidal sphere suspensions with newtonian matrices," J. Rheol. 57, 493-510 (2013).

[8] Didier Lootens, Henri Van Damme, Yacine Hémar, and Pascal Hébraud, "Dilatant flow of concentrated suspensions of rough particles," Phys. Rev. Lett. 95, 268302 (2005).

[9] John R Royer, Daniel L Blair, and Steven D Hudson, "Rheological signature of frictional 
179 [10] Lilian C Hsiao, Safa Jamali, Emmanouil Glynos, Peter F Green, Ronald G Larson, and

204 [21] Ryohei Seto and Giulio G Giusteri, "Normal stress differences in dense suspensions," J. Fluid.

$205 \quad$ Mech 857, 200-215 (2018).

206 [22] Frédéric Blanc, Elisabeth Lemaire, Alain Meunier, and François Peters, "Microstructure in 207 sheared non-Brownian concentrated suspensions," J. Rheol. 57, 273-292 (2013).

208 [23] H. Wilson and R. Davis, "Shear stress of a monolayer of rough spheres ? corrigendum," J. Michael J Solomon, "Rheological state diagrams for rough colloids in shear flow," Phys. Rev. Lett. 119, 158001 (2017).

[11] Matthieu Wyart and ME Cates, "Discontinuous shear thickening without inertia in dense non-Brownian suspensions," Phys. Rev. Lett. 112, 098302 (2014).

[12] Frédéric Blanc, François Peters, and Elisabeth Lemaire, "Local transient rheological behavior of concentrated suspensions," J. Rheol. 55, 835-854 (2011).

[13] Christopher Ness and Jin Sun, "Two-scale evolution during shear reversal in dense suspensions," Phys. Rev. E 93, 012604 (2016).

[14] François Peters, Giovanni Ghigliotti, Stany Gallier, Frédéric Blanc, Elisabeth Lemaire, and Laurent Lobry, "Rheology of non-Brownian suspensions of rough frictional particles under shear reversal: A numerical study," J. Rheol. 60, 715-732 (2016).

[15] J.J.J. Gillissen and H. J. Wilson, "Modeling sphere suspension microstructure and stress," Phys. Rev. E 98, 033119 (2018).

of deformable particles," J. Non-Newtonian Fluid Mech. 2, 169-189 (1977).

95 [17] B H A A Van den Brule and R J J Jongschaap, "Modeling of concentrated suspensions," J. Stat. Phys. 62, 1225-1237 (1991).

97 [18] Nhan Phan-Thien, "Constitutive equation for concentrated suspensions in Newtonian liquids," J. Rheol. 39, 679-695 (1995).

[19] R C Ball and John R Melrose, "A simulation technique for many spheres in quasi-static motion under frame-invariant pair drag and Brownian forces," Physica A 247, 444-472 (1997).

[20] E J Hinch and L G Leal, "Constitutive equations in suspension mechanics. Part 2. Approximate forms for a suspension of rigid particles affected by Brownian rotations," J. Fluid Mech. 76, 187-208 (1976). 
Fluid Mech. 814, 614-617 (2017). 